\title{
The origin of the lattice thermal conductivity enhancement at the ferroelectric phase transition in GeTe
}

\author{
Đorđe Dangić $\mathbb{D}^{1,2 凶}$, Olle Hellman ${ }^{3}$, Stephen Fahy ${ }^{1,2}$ and Ivana Savić $\mathbb{D}^{2 凶}$
}

The proximity to structural phase transitions in IV-VI thermoelectric materials is one of the main reasons for their large phonon anharmonicity and intrinsically low lattice thermal conductivity $\kappa$. However, the $\kappa$ of GeTe increases at the ferroelectric phase transition near $700 \mathrm{~K}$. Using first-principles calculations with the temperature dependent effective potential method, we show that this rise in $\kappa$ is the consequence of negative thermal expansion in the rhombohedral phase and increase in the phonon lifetimes in the high-symmetry phase. Strong anharmonicity near the phase transition induces non-Lorentzian shapes of the phonon power spectra. To account for these effects, we implement a method of calculating $\kappa$ based on the Green-Kubo approach and find that the Boltzmann transport equation underestimates $\kappa$ near the phase transition. Our findings elucidate the influence of structural phase transitions on $\kappa$ and provide guidance for design of better thermoelectric materials.

npj Computational Materials (2021)7:57; https://doi.org/10.1038/s41524-021-00523-7

\section{INTRODUCTION}

Reducing the lattice thermal conductivity $k$ is one of the most successful ways of improving the efficiency of thermoelectric materials $^{1-7}$. Many of the best thermoelectric materials have intrinsically low lattice thermal conductivity. This is usually related to the large anharmonicity of phonon modes, which can stem from weak bonding, as in van der Waals materials ${ }^{2,5,8-11}$, or rattling modes ${ }^{12-17}$. Another source of large anharmonicity in good thermoelectric materials can be the proximity to structural phase transitions, as in the case of IV-VI materials ${ }^{18-24}$. For example, germanium telluride (GeTe) undergoes the ferroelectric phase transition at $\sim 700 \mathrm{~K}$ and has intrinsically low lattice thermal conductivity and high thermoelectric efficiency ${ }^{25-27}$.

Recent computational work has predicted that driving IV-VI materials closer to the ferroelectric phase transition via strain or alloying can lead to a drastically lower lattice thermal conductivity $^{22,23}$. Under the assumption of the displacive phase transition, it was found that coupling between soft transverse optical (TO) modes and the heat-carrying acoustic modes is the main reason for the $\kappa$ reduction. At the displacive transition, the frequency of the soft TO mode collapses, becoming effectively zero. Since scattering rates are inversely proportional to phonon frequencies, the lifetimes of the acoustic modes that couple to soft TO modes decrease dramatically, leading to a considerable $\kappa$ reduction ${ }^{22,23}$.

Surprisingly, experimental studies have shown that the lattice thermal conductivity increases at the ferroelectric phase transition in $\mathrm{GeTe}^{25-32}$. This is at odds with measurements in some other materials going through ferroelectric phase transitions, where a significant decrease in $K$ is observed ${ }^{33}$. The reason for the anomalous behaviour of $k$ at the phase transition in GeTe remains unknown. Understanding the microscopic origin of the $K$ increase at the ferroelectric phase transition in GeTe may lead to the design of improved thermoelectric materials.

Here, we study how driving GeTe near the ferroelectric phase transition via temperature affects its lattice thermal conductivity, making no assumptions about the nature of the phase transition. Unlike the previous work ${ }^{22,23}$, we calculate interatomic force constants at different temperatures using the state-of-the-art, temperature-dependent effective potentials (TDEP) method ${ }^{34-36}$. We find that the increase of the lattice thermal conductivity of GeTe at the phase transition in the rhombohedral phase comes from the negative thermal expansion that enhances the phonon group velocities. In the cubic phase, the phonon lifetimes increase, leading to an even more substantial increase of $\kappa$. Large anharmonicity of phonon modes minimises the phonon lifetimes at the phase transition in the rhombohedral phase and leads to non-Lorentzian power spectra of phonon modes. We implement a method of calculating $\kappa$ that includes these non-Lorentzian lineshapes of the phonon power spectra near the phase transition. This approach further increases $K$ at the phase transition, which can be attributed to further softening of the phonon frequencies due to phonon-phonon interaction.

\section{RESULTS AND DISCUSSION}

\section{TO mode softening at the phase transition}

GeTe is a ferroelectric material, exhibiting a spontaneous polarisation below $600-700 \mathrm{~K}^{37-40}$ (the critical temperature strongly depends on the free charge carrier concentration). This occurs due to a slight offset of the Te sublattice along with one of the body diagonals of the rocksalt structure. At temperatures higher than $600-700 \mathrm{~K}$, GeTe transforms to the rocksalt structure, losing its ferroelectric nature ${ }^{41-44}$. Below $600-700 \mathrm{~K}$, the GeTe structure can be described by the following set of lattice vectors

$$
\begin{aligned}
& \mathbf{r}_{1}=a(b, 0, c), \\
& \mathbf{r}_{2}=a\left(-\frac{b}{2}, \frac{b \sqrt{3}}{2}, c\right), \\
& \mathbf{r}_{3}=a\left(-\frac{b}{2},-\frac{b \sqrt{3}}{2}, c\right),
\end{aligned}
$$

where $a$ is the lattice constant, $b=\sqrt{2(1-\cos \theta) / 3}$, $c=\sqrt{(1+2 \cos \theta) / 3}$, and $\theta$ is the angle between the primitive lattice vectors. The atomic positions in this structure are taken to be: $\mathrm{Ge}(0.0,0.0,0.0)$ and $\mathrm{Te}(0.5+\mu, 0.5+\mu, 0.5+\mu)$ in reduced coordinates. If the phase transition is displacive, as assumed in

${ }^{1}$ Department of Physics, University College Cork, Cork, Ireland. ${ }^{2}$ Tyndall National Institute, Cork, Ireland. ${ }^{3}$ Department of Physics, Chemistry and Biology (IFM), Linköping University, Linköping, Sweden. ${ }^{\circledR}$ email: djordje.dangic@tyndall.ie; ivana.savic@tyndall.ie 

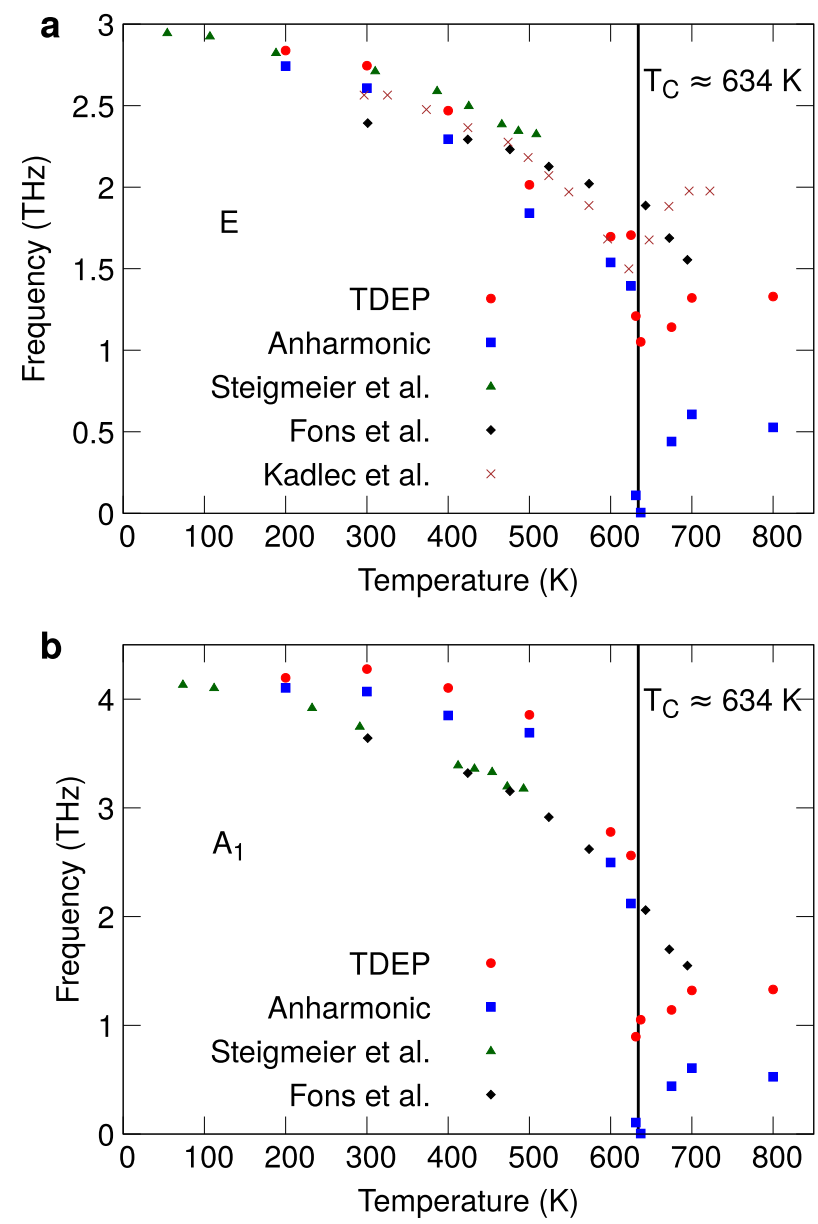

Fig. 1 Temperature dependence of the zone centre optical phonon frequencies. a The lower transverse optical (TO) mode $(E)$ and $\mathbf{b}$ the higher TO mode $\left(A_{1}\right)$ at the zone centre as we approach $\mathbf{q}$ $=0$ along the $X-\Gamma$ direction, perpendicular to the trigonal axis. Red circles and blue squares represent the calculated temperaturedependent effective potential (TDEP) and anharmonic phonon frequencies, while other symbols represent the experimental results from refs. $43,45,48$. The TDEP frequency is the square root of the eigenvalue of the dynamical matrix at a particular temperature, while the anharmonic frequency is the peak of the phonon mode power spectrum. The black vertical line represents the phase boundary between the rhombohedral and rocksalt structures in our calculations $(\approx 634 \mathrm{~K})$.

refs. ${ }^{22,23}$, the angle $\theta$ becomes $60^{\circ}$ at the phase transition, while the interatomic displacement parameter $\mu$ becomes zero ${ }^{41,42}$. In this type of phase transition, the TDEP frequency of the soft mode also collapses to zero ${ }^{45,46}$. Here, we define the TDEP frequency of the phonon mode as the square root of the eigenvalue of the dynamical matrix for that phonon mode at a certain temperature. In the order-disorder phase transition, both the TDEP frequency of the soft mode and the local interatomic displacement are nonzero $^{43,44,47}$. It is still under debate which type of phase transition occurs in GeTe $\mathrm{G1}^{41-45}$.

We have calculated the phonon dispersion of GeTe at different temperatures using the TDEP method and neglecting longitudinal optical (LO)/TO splitting due to high hole concentration in experimental GeTe samples. The TDEP method allows us to calculate TDEP frequencies of phonon modes at different temperatures. In these calculations, we used the structural parameters calculated at appropriate temperatures (see the "Methods" section). Figure 1 shows the TDEP frequencies of the two TO modes at the Brillouin zone centre in GeTe as we approach
$\Gamma$ from the $\Gamma-X$ direction (corresponding to the non-degenerate $A_{1}$ mode and the $E$ mode which is degenerate with the longitudinal optical (LO) mode). The phase transition temperature obtained in our calculations is $T_{\mathrm{C}} \approx 634 \mathrm{~K}$ (see "Methods"). The TDEP frequencies of both phonon modes are strongly renormalized by anharmonic interaction and lattice thermal expansion. The TDEP frequencies of these phonon modes soften drastically at the phase transition, but they do not become zero, indicating that the phase transition in GeTe might not be of the displacive type.

The observed phonon frequency in experiments is not the TDEP frequency, but what we will call in the rest of the paper the anharmonic frequency i.e. the peak of the phonon mode power spectrum. Blue squares in Fig. 1 represent the computed anharmonic frequencies of optical modes at the zone centre, which do fall to zero at the phase transition, in contrast to the TDEP frequencies. Our results thus suggest that the observation of phonon mode softening is not conclusive proof of the displacive type of the phase transition, as previously argued in the case of $\mathrm{GeTe}^{45}$. The calculated phonon frequencies (both TDEP and anharmonic) are very similar in the two different phases for the temperatures closest to the phase transition (at 631 and $637 \mathrm{~K}$ ).

Our computed anharmonic optical frequencies at the zone centre agree fairly well with those measured in experiments, see Fig. 1. This agreement highlights the accuracy of the TDEP method even for the challenging cases of materials undergoing structural phase transitions. We note that the critical temperature in ref. ${ }^{45}$ is around $600 \mathrm{~K}$, in ref. ${ }^{43}$ approximately $700 \mathrm{~K}$ and in ref. ${ }^{48} 650 \mathrm{~K}$. This difference in the calculated and measured critical temperature is expected since the critical temperature strongly depends on the number of free charges carriers ${ }^{49,50}$.

\section{Phonon spectral function}

Harmonic (and/or TDEP) frequencies are a valid description of lattice dynamics only in the absence of phonon-phonon interaction. In inelastic neutron scattering experiments that measure phonon spectral functions, harmonic phonons would produce zero linewidth signals, revealing infinitely long lived quasiparticles. However, in real materials phonons interact with each other, thus broadening phonon spectral functions, with linewidths inversely proportional to phonon lifetimes. This effect can be described using the concept of phonon self-energy that quantifies the strength of phonon-phonon interaction ${ }^{51-53}$.

The probability of an incoming neutron to interact with a phonon system acquiring/losing energy $\hbar \Omega$ and momentum $\hbar \mathbf{q}$ is proportional to the spectral function ${ }^{51-54}$

$$
s(\mathbf{q}, \Omega) \sim \sum_{s}\left\langle u_{\mathbf{q}, s}^{\dagger} u_{\mathbf{q}, s}\right\rangle=\sum_{s} \frac{4 \omega_{\mathbf{q}, s}^{2}}{\pi\left(\mathrm{e}^{\beta h \Omega}-1\right)} \frac{\Gamma_{\mathbf{q}, s}(\Omega)}{\left(\Omega^{2}-\omega_{\mathbf{q}, s}^{2}-2 \omega_{\mathbf{q}, s} \Delta_{\mathbf{q}, s}(\Omega)\right)^{2}+4 \omega_{\mathbf{q}, s}^{2} \Gamma_{\mathbf{q}, s}^{2}(\Omega)},
$$

where $u_{\mathbf{q}, s}$ is the phonon displacement operator, $\omega_{\mathbf{q}, s}$ is the TDEP frequency of the phonon mode with wave vector $\mathbf{q}$ and phonon branch $s, \beta$ is $1 / k_{B} T$ with $k_{B}$ being the Boltzmann constant and $T$ the temperature, and $\hbar$ is the reduced Planck constant. $\Delta_{\mathbf{q}, s}$ and $\Gamma_{\mathbf{q}, s}$ are the real and imaginary part of the phonon-self energy, respectively. In a weakly anharmonic material, this expression can be reduced to a Lorentzian with a half-width of $\Gamma_{\mathbf{q}, s}$ and the position of the peak at $\omega_{\mathbf{q}, s}+\Delta_{\mathbf{q}, s}$. In this case, the phonon lifetime can be calculated as $\tau_{\mathbf{q}, s}=\frac{1}{2 \Gamma_{\mathbf{q} s}}$. However, in materials with strong anharmonicity, phonon spectral functions can exhibit exotic behaviour with satellite peaks, shoulders, etc. ${ }^{18,20,55-58}$.

First we show the phonon spectral function of GeTe near the phase transition in the rhombohedral phase $(631 \mathrm{~K})$ along a high symmetry path, see Fig. 2. The black lines represent the TDEP frequencies $\omega_{\mathbf{q}, s}$ calculated at $631 \mathrm{~K}$ and the cyan dashed lines are the TDEP frequencies at $300 \mathrm{~K}$. The TDEP frequencies of optical modes soften from 300 to $631 \mathrm{~K}$. The acoustic mode frequencies soften as well in the $F-\Gamma$ and the in-plane $(\Gamma-X)$ directions. This is 


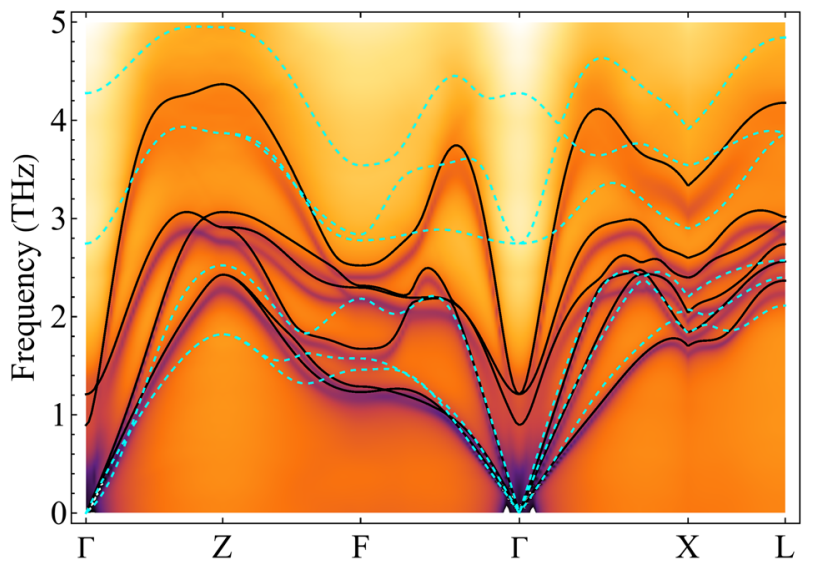

Fig. 2 Phonon spectral function along high symmetry lines in GeTe at the phase transition. The spectral function is calculated using Eq. (2). Black lines represent the temperature-dependent effective potential (TDEP) frequencies calculated at $631 \mathrm{~K}$ (close to the critical temperature $634 \mathrm{~K}$ ) and dashed cyan lines are the TDEP phonon band structure at $300 \mathrm{~K}$. The yellow colour corresponds to the lower, while the purple colour corresponds to the higher value of the phonon spectral function.

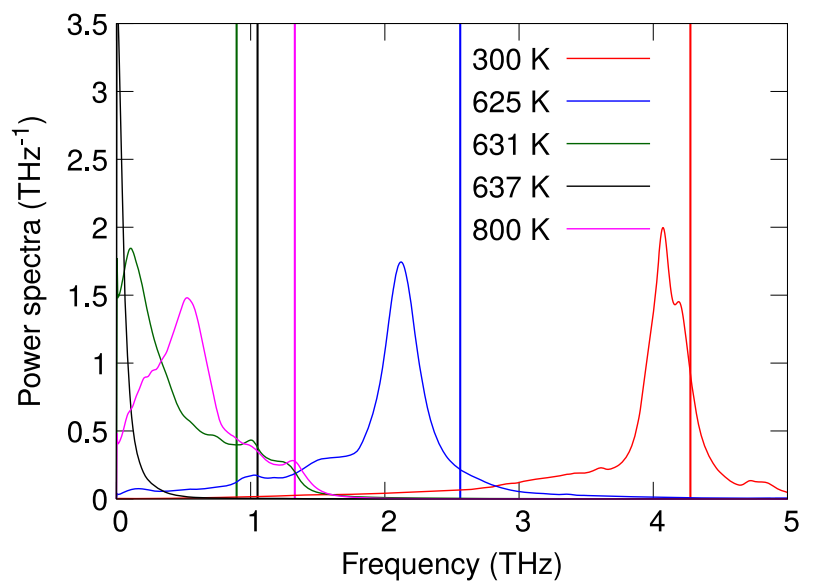

Fig. 3 Spectral function for the zone centre $A_{1}$ mode in GeTe at different temperatures. Vertical lines represent the values of the TDEP $A_{1}(\Gamma)$ frequencies at different temperatures. A drastic deviation from the Lorentzian shape is evident for the entire temperature range and most prominent in the vicinity of the phase transition $(634 \mathrm{~K})$.

the consequence of the overall softening of the second-order force constants with temperature. On the other hand, the acoustic modes along the $\Gamma-Z$ direction (the direction along the trigonal axis) stiffen because of the negative thermal expansion, as we will discuss in the next section. The spectral function shows further softening of the phonon frequencies due to anharmonic phonon-phonon interaction. As expected, the optical phonons at $\Gamma$ can not be clearly resolved in the graph of the phonon spectral function. Supplementary Note 1 shows the phonon band structure in the cubic phase near the phase transition, at $637 \mathrm{~K}$.

Figure 3 shows the unusual features of the spectral function for the $A_{1}$ mode of GeTe at the zone centre for several different temperatures. A non-Lorentzian behaviour of the phonon spectral function is evident even at $300 \mathrm{~K}$, very far from the phase transition. The broadening of the power spectrum is large, revealing the short lifetime of this phonon mode. The distortion of the power spectrum is stronger at $625 \mathrm{~K}$, with a very large shift

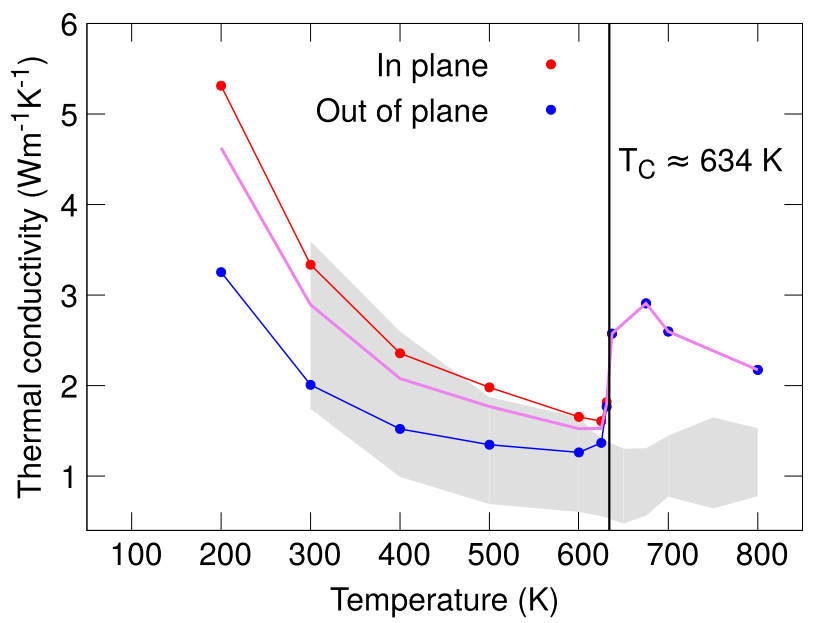

Fig. 4 Temperature dependence of the lattice thermal conductivity of GeTe. Red and blue lines represent the calculated values using the Boltzmann transport approach in the directions perpendicular and parallel to the trigonal [111] axis, respectively. The purple line represents the average values of the computed lattice thermal conductivity. The grey region represents the experimental results collected from refs. ${ }^{25-32}$.

of the peak of the spectral function compared to the TDEP frequency. For temperatures near the phase transition, the power spectrum peaks around $0 \mathrm{THz}$ as expected at the phase transition (see Fig. 1). At temperatures higher than the phase transition temperature, the peak of the power spectrum is at nonzero frequencies but is still strongly renormalized compared to the TDEP frequency.

Non-Lorentzian shapes of the phonon power spectra of the optical modes in GeTe at the phase transition are the consequence of the coupling of these phonon modes to the entire phonon bath, rather than coupling to specific phonons. We test this by calculating the power spectrum of the $A_{1}$ phonon mode disregarding the coupling to specific phonon branches. We find that the change in the power spectrum does not substantially vary depending on which phonon branch we disregard. Similar behaviour can be seen in the spectral function of the $E$ mode (see Supplementary Note 2).

\section{Lattice thermal conductivity in the Boltzmann transport approach}

Next, we calculate the lattice thermal conductivity of GeTe for a range of temperatures including both rhombohedral and rocksalt phases (see Fig. 4), combining the TDEP method with the Boltzmann transport approach. Overall, the lattice thermal conductivity is inversely proportional to temperature, as a result of the linear dependence of phonon populations with a temperature above the Debye temperature (200 K for GeTe). The calculated $\kappa$ deviates from the $1 / T$ law near the phase transition, where there is a large $k$ increase at the phase transition and in the cubic phase. At high temperatures, the $\kappa$ in the cubic phase regains the $1 / T$ dependence.

There is an anisotropy in the lattice thermal conductivity in the rhombohedral phase (Fig. 4), as a consequence of the van der Waals gaps formed due to the Te sublattice offset. The direction perpendicular to the van der Waals gaps (i.e., parallel to the trigonal [111] axis) has weaker bonding, leading to the lower phonon group velocities and $k$ in that direction. Anisotropy of the lattice thermal conductivity disappears close to the phase transition and is not present in the rocksalt phase.

The agreement between the computed and experimental $\kappa$ values is very good in the whole temperature range of the 

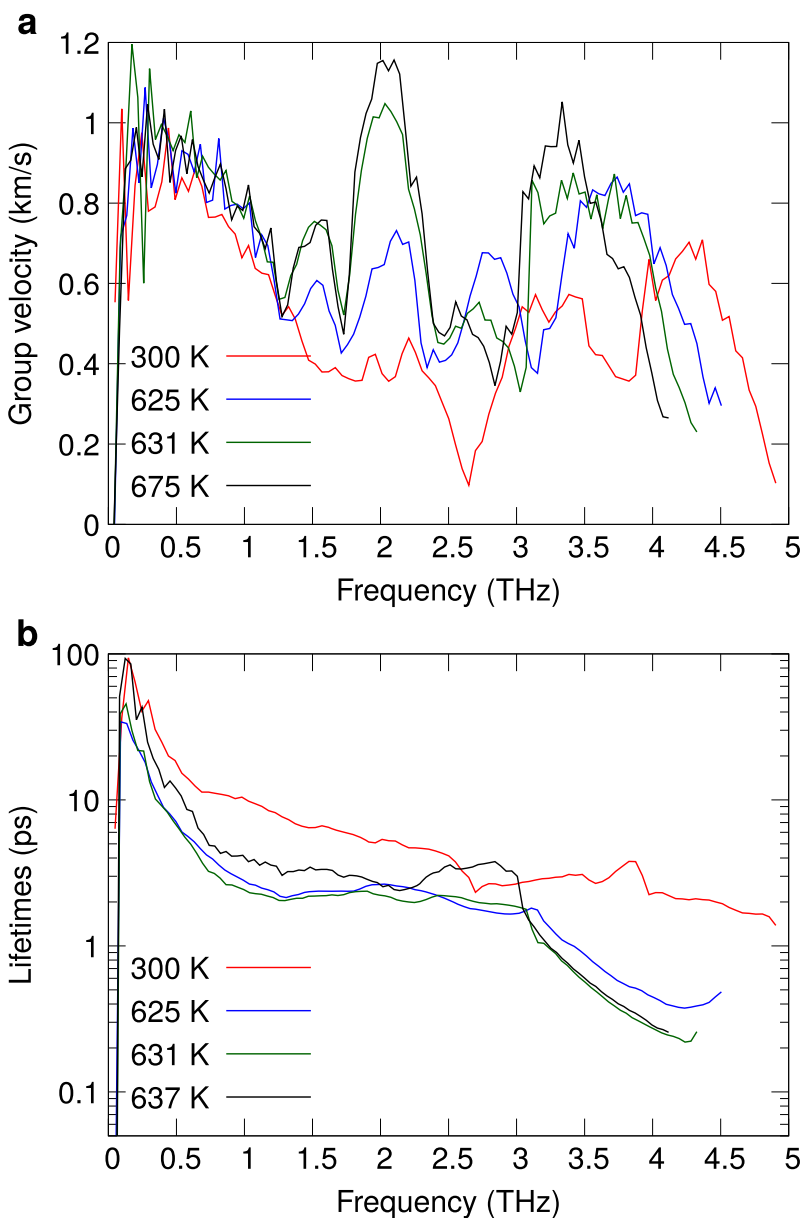

Fig. 5 Frequency dependence of phonon transport properties. a Average phonon group velocities and $\mathbf{b}$ average phonon lifetimes of GeTe vs. phonon frequency for different temperatures. Averaging is carried out by convolving the calculated values of these quantities with a Gaussian (see Eq. (3)).

rhombohedral phase, especially if we consider the average $\kappa$ (purple line in Fig. 4). Almost all experiments show an increase in the lattice thermal conductivity in the vicinity of phase transition ${ }^{25-32}$, similarly to our results. The discrepancy between our results and experiments increases as we get closer to the phase transition and particularly in the cubic phase. Our results consistently overestimate $\kappa$ compared to experiments. In the rhombohedral phase, we would expect scattering from lattice imperfections, such as ferroelectric domain walls ${ }^{59-63}$, to further reduce the computed lattice thermal conductivity, but this is not the case in the cubic phase.

The differences between our calculated $\kappa$ values and experiments could also arise from the fact that GeTe has a large number of Ge vacancies. Several recent publications that report calculations of $k$ in GeTe stress the importance of including point defect scattering in the calculation ${ }^{24,64}$. Additionally, the experimental investigation of the phase transition in $\mathrm{GeTe}^{65}$ noted a huge increase of Ge vacancies in the cubic phase. These point defects scatter higher frequency phonons more effectively ${ }^{66}$. Considering this and the fact that the main contribution to the lattice thermal conductivity shifts to the phonons with higher frequencies at higher temperatures (see Supplementary Note 3), we conclude that the lack of point defect scattering in our calculations might be one of the possible reasons for the larger discrepancy in $\kappa$ in the cubic phase between our calculated values and experiments.
The discrepancies between theory and experiment could also stem from omitting the higher-order terms in the Taylor expansion of the interatomic forces (we include the second and third-order terms only). However, to the lowest approximation, the fourth-order anharmonic terms would only affect the real part of the self-energy ${ }^{52}$ and not the imaginary part, which would mean that the phonon lifetimes should remain unchanged. In addition, since the force constants in TDEP are obtained through a fitting procedure, the fourth-order force constants should be smaller than the third-order ones. However, a more detailed study is needed to completely resolve this issue.

Experimental values of lattice thermal conductivity are usually extracted from the total thermal conductivity measurements using the Wiedemann-Franz law to eliminate the electronic contribution to the thermal conductivity, whose validity at structural phase transitions is not well understood. In addition, most references use the single parabolic band Kane model to extract the Lorenz factor from measurements of the Seebeck coefficient, which is not appropriate in GeTe due to the intrinsically complicated Fermi surface $^{31}$. Such lattice thermal conductivity values can differ widely near structural phase transitions, and sometimes an increase in the total thermal conductivity is assigned to the electronic contribution. Here, we show that the increase in the thermal conductivity of GeTe at the phase transition, at least partially, comes from the lattice thermal conductivity. The difference between our theoretical and experimental results in the cubic phase might partially be due to an inaccurate estimation of the electronic contribution to the total thermal conductivity in experiments. Measuring the thermal conductivity of GeTe in an applied magnetic field (to exclude the electronic thermal conductivity) would test our predictions of the increased lattice thermal conductivity at the phase transition.

To understand the anomalous behaviour of $\kappa$ near the phase transition, we calculate the average phonon lifetimes and group velocities at different temperatures, Fig. 5. For example, the average values of the phonon lifetimes in the vicinity of the phonon frequency $\omega_{0}$ are given as

$$
\bar{\tau}\left(\omega_{0}\right)=\frac{\sum_{\lambda} \tau\left(\omega_{\lambda}\right) \exp \left(-\frac{\left(\omega_{0}-\omega_{\lambda}\right)^{2}}{\sigma^{2}}\right)}{\sum_{\lambda} \exp \left(-\frac{\left(\omega_{0}-\omega_{\lambda}\right)^{2}}{\sigma^{2}}\right)},
$$

where the sum goes over all phonon modes $\lambda$, and $\sigma$ is the smearing parameter taken to be $\sigma=\omega_{\mathrm{D}} /(N+1)$, where $\omega_{\mathrm{D}}$ is the Debye frequency and $N$ is the number of $\omega_{0}$ frequencies.

The phonon group velocities of GeTe are mostly independent of temperature, except very close to the phase transition, see Fig. $5 \mathrm{a}$. In this temperature region $(600-675 \mathrm{~K})$, there is an increase in the phonon group velocities across most of the frequency range and most noticeably for phonons between 1 and $3 \mathrm{THz}$. This is the frequency region that contributes most to the thermal conductivity (see Supplementary Note 3). We thus conclude that the anomalous increase of the thermal conductivity at the phase transition is partially due to this rise in the phonon group velocities. We find that the increase in group velocities originates from the lattice contraction near the critical temperature ${ }^{41,42,67}$. This can be understood from the observation that the increase of phonon group velocities happens most prominently along out of the plane direction, where negative thermal expansion occurs ${ }^{67}$.

Phonon lifetimes in weakly anharmonic materials usually follow the $1 / T$ law, similar to thermal conductivity. This is the case in our calculations in the rhombohedral phase far from the phase transition. At the phase transition, however, the phonon lifetimes of acoustic modes decrease more than expected from the $1 / T$ scaling. This is a signature of stronger anharmonicity of acoustic phonon modes closer to the phase transition in the rhombohedral phase. Optical modes have more complicated behaviour. While soft optical modes near the zone centre have much lower lifetimes at the phase transition, this is not the case for the two lowest 
optical modes in the rest of the Brillouin zone. Even more intriguing is the behaviour of the highest frequency optical modes. At low temperatures, they usually have frequencyindependent lifetimes, but at the phase transition, they decrease exponentially with frequency. The calculated phonon linewidths of the zone centre phonon modes are the same order of magnitude as the measured ones ${ }^{45}$ (see Supplementary Note 4).

In the cubic phase, there is a substantial increase in the phonon lifetimes with respect to the rhombohedral phase (see Fig. 5b). The phonon lifetimes at $637 \mathrm{~K}$ are larger in most of the frequency range compared to the temperatures closest to the phase transition in the rhombohedral phase $(625$ and $631 \mathrm{~K})$. Interestingly, the phonon lifetimes at $675 \mathrm{~K}$ are larger than the phonon lifetimes at $300 \mathrm{~K}$ rescaled by temperature (i.e. by $675 \mathrm{~K} / 300 \mathrm{~K}$ ), revealing stronger intrinsic anharmonicity of the rhombohedral phase. Third-order force constants are much stronger in the rhombohedral phase, even for very similar temperatures and structures (631 vs. $637 \mathrm{~K}$ ), see Supplementary Note 5.

In conclusion, the increase of the lattice thermal conductivity near the phase transition can be attributed to two phenomena, depending on the structure of GeTe. In the rhombohedral phase, the increase in the thermal conductivity is due to the negative thermal expansion that causes phonon group velocities to increase, increasing phonon mean free paths. On the other hand, in the cubic phase, phonon lifetimes increase dramatically due to the lower intrinsic anharmonicity of this high-symmetry phase.

\section{Lattice thermal conductivity using the Green-Kubo method}

The non-Lorentzian behaviour of the phonon spectral function raises the question of whether the Boltzmann transport equation employed in the calculation of the $k$ values in Fig. 4 is valid close to the phase transition. It is assumed in the derivation of the Boltzmann equation that phonons are well-defined quasiparticles with unique frequencies and lifetimes. This implies that their spectral weights are the Lorentzian functions centred at the harmonic or TDEP frequencies and the widths equal to the phonon lifetimes. However, evidently, this does not hold close to the phase transition, see Fig. 3.

We include the effect of non-Lorentzian lineshapes on lattice thermal conductivity following the Green-Kubo approach derived in refs. ${ }^{68,69}$. In this approach, the heat current in the Cartesian direction $j$ is defined $a s^{68,70,71}$

$j^{j}(t)=\frac{1}{2 N V} \sum_{\mathbf{q}, s, s^{\prime}} \hbar \omega_{\mathbf{q}, s} p_{\mathbf{q}, s}(t) u_{\mathbf{q}, s^{\prime}}^{\dagger}(t) v_{\mathbf{q}, s, s^{\prime}}^{j}$

where $v_{\mathbf{q}, s}^{j}$ is the group velocity of the phonon mode with wave vector $\mathbf{q}$ and branch $s, V$ is the volume of the unit cell, $N$ is the number of $\mathbf{q}$ points, and $p_{\mathbf{q}, s}(t)$ and $u_{\mathbf{q}, s}(t)$ are the Fourier transforms of the phonon momentum and position operators. The lattice thermal conductivity tensor is obtained by employing the Green-Kubo relation ${ }^{72}$

$\kappa^{i, j}=\frac{N V}{k_{B} T^{2}} \Re \int\left\langle J^{i}(0) j^{j}(t)\right\rangle \mathrm{d} t$.

The spectral theorem is then used to relate the heat current autocorrelation function in the equation above to the one-particle retarded Green function ${ }^{51,69,73-75}$. The final expression for the thermal conductivity in this approach is ${ }^{68,69}$

$$
\begin{gathered}
\kappa^{i, j}=4 \frac{\hbar^{2} k \beta^{2}}{N V \pi} \sum_{\mathbf{q}, s, s^{\prime}} \omega_{\mathbf{q}, s}^{2}\left(\omega_{\mathbf{q}, s}^{2}+\omega_{\mathbf{q}, s^{\prime}}^{2}\right) v_{\mathbf{q}, s, s^{\prime}}^{i} v_{\mathbf{q}, s, s^{\prime}}^{j} \int_{-\infty}^{\infty} \mathrm{d} \Omega \frac{\exp (\beta \hbar \Omega)}{(\exp (\beta \hbar \Omega)-1)^{2}} \\
\times \frac{\Omega^{2} \Gamma_{\mathbf{q}, s}(\Omega) \Gamma_{\mathbf{q}, s^{\prime}}(\Omega)}{\left[\epsilon_{\mathbf{q}, s}^{2}+4 \omega_{\mathbf{q}, s}^{2} \Gamma_{\mathbf{q}, s^{2}}^{2}(\Omega)\right]\left[\epsilon_{\mathbf{q}, s^{\prime}}^{2}+4 \omega_{\mathbf{q}, s^{\prime}}^{2} \Gamma_{\mathbf{q}, s^{\prime}}^{2}(\Omega)\right]},
\end{gathered}
$$
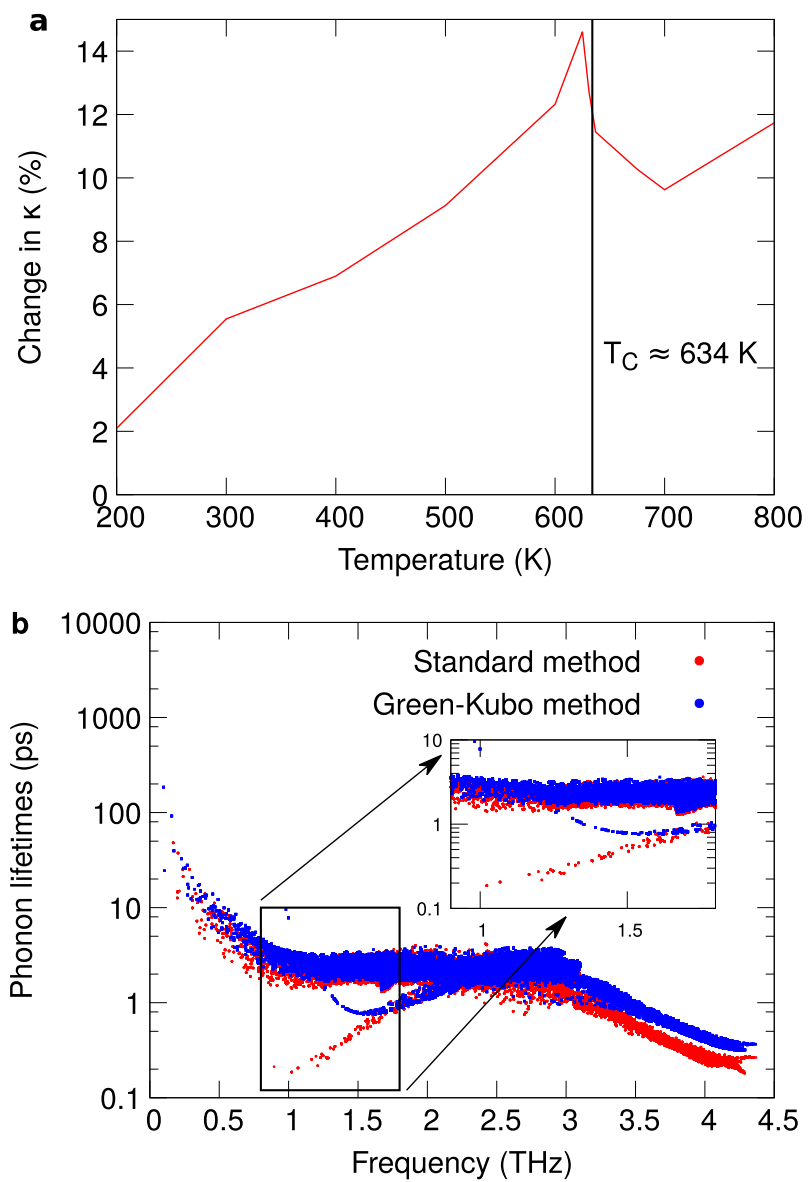

Fig. 6 Green-Kubo lattice thermal conductivity. a Difference in the calculated lattice thermal conductivity using the Green-Kubo method (Eq. (6)) and the Boltzmann transport equation. b Phonon lifetimes of GeTe (see text for explanation) in the Green-Kubo and Boltzmann transport equation approach plotted vs. TDEP frequency of phonon modes. Inset shows the region of soft phonon modes where the change is most noticeable.

where $\epsilon_{\mathbf{q}, s}$ is

$\epsilon_{\mathbf{q}, s}=\Omega^{2}-\omega_{\mathbf{q}, s}^{2}-2 \omega_{\mathbf{q}, s} \Delta_{\mathbf{q}, s}(\Omega)$.

$\mathbf{v}_{\mathbf{q}, s, s^{\prime}}$ is the generalised phonon group velocity ${ }^{70}$

$\mathbf{v}_{\mathbf{q}, s, s^{\prime}}=\frac{\mathrm{i}}{2 \sqrt{\omega_{\mathbf{q}, s} \omega_{\mathbf{q}, s^{\prime}}}} \sum_{a, b} \mathbf{X}_{\mathbf{q}, s}^{a} \sum_{\mathbf{R}}\left(\mathbf{R} \frac{\Phi_{a, b}(\mathbf{R})}{\sqrt{m_{a} m_{b}}}\right) \mathbf{x}_{\mathbf{q}, s^{\prime}}^{b}$,

where $\mathbf{X}_{\mathbf{q}, s}$ is the eigenvector of the phonon with wave vector $\mathbf{q}$ and branch $s, \Phi_{a, b}(\mathbf{R})$ is the force constant between atoms with masses $m_{a}$ and $m_{b}$ and the vector distance $\mathbf{R}$.

We can separate Eq. (6) into two parts. The first part is diagonal $\left(s=s^{\prime}\right)$. In the limit of small anharmonicity $\left(\Delta_{\mathbf{q}, s}=0\right.$ and $\left.\Gamma_{\mathbf{q}, s} \ll \omega_{\mathbf{q}, s}\right)$, this part reduces to the standard solution of the Boltzmann equation in the relaxation time approximation. The second, nondiagonal part $\left(s \neq s^{\prime}\right)$, can be reduced in the limit of small anharmonicity to the expressions similar to the ones given in refs. ${ }^{76,77}$. The non-diagonal contribution to the lattice thermal conductivity will become prominent only if there is a substantial overlap in the spectral functions of two phonon modes with the same wave vector. This is only true in the case of strong anharmonicity or when spectral functions broaden due to disorder.

We have implemented the expression given by Eq. (6) with the TDEP method. Figure 6 a shows the difference between our results obtained using Eq. (6) and BTE (Fig. 4). We can see that the BTE underestimates the thermal conductivity in the whole 
temperature range. In addition, we can see that the underestimation is not large, around $10 \%$ even at high temperatures. Overall, the difference scales linearly with temperature. We can also see that the difference is largest at the phase transition, which is expected considering large deviations from the Lorentzian shape of the phonon spectral functions in this region (see Fig. 2). The contribution of the non-diagonal part of the lattice thermal conductivity is comparable to the overall enhancement of the diagonal part due to non-Lorentzian shapes of the phonon spectral functions.

To understand the reason for the increased difference in $K$ at the phase transition obtained by the standard BTE method and using the Green-Kubo relation (Eq. (6)), we show the phonon lifetimes of GeTe calculated using the two methods in Fig. 6b. We define the phonon lifetimes in the Green-Kubo method as

$$
\tau_{\mathbf{q}, s}^{G K}=8 \frac{\hbar^{2} k_{B} \beta^{2} \omega_{\mathbf{q}, s}^{4}}{\pi c_{\mathbf{q}, s}} \int_{-\infty}^{\infty} \mathrm{d} \Omega \frac{\exp (\beta \hbar \Omega)}{(\exp (\beta \hbar \Omega)-1)^{2}} \frac{\Omega^{2} \Gamma_{\mathbf{q}, s}^{2}(\Omega)}{\left[\epsilon_{\mathbf{q}, s}^{2}+4 \omega_{\mathbf{q}, s}^{2} \Gamma_{\mathbf{q}, s}^{2}(\Omega)\right]^{2}},
$$

where $c_{\mathbf{q}, s}$ is the harmonic heat capacity of the phonon mode $(\mathbf{q}, \mathbf{s})$. The increase of the phonon lifetimes in the Green-Kubo method is visible in the whole Brillouin zone. It is, however, most prominent in the region of soft phonon modes, where the phonon lifetimes increase by a factor of 100 . The increase in phonon lifetimes mostly comes from the shifts of the peaks of the spectral functions, which increases the heat capacity of the phonon modes compared to the harmonic heat capacity.

\section{DISCUSSION}

Here, we highlight the advantages of the Green-Kubo method we implemented here over the standard approaches for computing $k$ in strongly anharmonic materials. Unlike the Boltzmann transport equation, the Green-Kubo method accounts for non-Lorentzian shapes of phonon spectral functions. It is possible to include these effects also by running long molecular dynamics (MD) simulations. Compared to MD, the Green-Kubo method presented here is faster and easier to converge, which is particularly important if these methods are combined with first-principles calculations. The results obtained using this approach are easier to interpret and analyse compared to traditional MD approaches. Unlike MD simulations, the Green-Kubo method uses the Bose-Einstein statistics for phonons. In addition, this method gives the nondiagonal part of lattice thermal conductivity, accounting explicitly for the whole phonon power spectra. The method of refs. ${ }^{76,77}$ includes only the values of the phonon self-energy at the harmonic frequency in the evaluation of the non-diagonal contribution to $k$ and is not applicable to strongly anharmonic materials.

In conclusion, we have performed a detailed first-principles study of the lattice thermal conductivity $k$ of GeTe close to the ferroelectric phase transition. The TDEP frequencies of the soft modes, although dramatically softened, do not become zero at the phase transition. On the other hand, strong anharmonicity causes the spectral functions of the soft modes to collapse and effectively peak at zero frequency. Strong anharmonicity minimises the acoustic phonon modes lifetimes at the phase transition. However, we calculate an increase in the lattice thermal conductivity at the phase transition, in agreement with experiments. In the rhombohedral phase, this effect is due to the negative thermal expansion that increases phonon group velocities. In the cubic phase, the increase in $k$ is primarily driven by increased phonon lifetimes due to the smaller anharmonicity of phonon modes compared to the rhombohedral phase. We implement a method to compute lattice thermal conductivity that includes the observed non-Lorentzian power spectra of phonon modes. Using this approach, we find that the calculated $k$ increases even further at the phase transition, which is the consequence of larger phonon populations due to softening of the phonon modes caused by phonon-phonon interaction.

\section{METHODS}

\section{Boltzmann transport equation}

We calculate the lattice thermal conductivity of GeTe from first principles using density functional theory (DFT) and the Boltzmann transport equation (BTE). In this approach, the lattice thermal conductivity tensor is given $a^{68}$

$\kappa^{i, j}=\frac{1}{N V} \sum_{\mathbf{q}, s} c_{\mathbf{q}, s} v_{\mathbf{q}, s}^{i} v_{\mathbf{q}, s}^{j} \tau_{\mathbf{q}, s}$,

where $i$ and $j$ are the Cartesian directions, $N$ is the number of $\mathbf{q}$ points, $V$ is the unit cell volume, $c_{\mathbf{q}, s}$ is the phonon mode heat capacity and $v_{\mathbf{q}, s}^{i}$ is the group velocity of the phonon mode $(\mathbf{q}, s)$ in the direction $i$. The relaxation time of the same mode is $\tau_{\mathbf{q}, s}=1 / 2 \Gamma_{\mathbf{q}, s}$, where the imaginary part of the phonon self-energy due to three-photon scattering is given $\mathrm{as}^{51}$

$$
\begin{aligned}
\Gamma_{\lambda}= & \frac{\pi \hbar}{16 N} \sum_{\lambda^{\prime}, \lambda^{\prime \prime}}\left|\Phi_{\lambda \lambda^{\prime} \lambda^{\prime \prime}}\right|^{2}\left\{\left(n_{\lambda^{\prime}}+n_{\lambda^{\prime \prime}}+1\right) \delta\left(\omega_{\lambda}-\omega_{\lambda^{\prime}}-\omega_{\lambda^{\prime \prime}}\right)\right. \\
& \left.+2\left(n_{\lambda^{\prime}}-n_{\lambda^{\prime \prime}}\right) \delta\left(\omega_{\lambda}-\omega_{\lambda^{\prime}}+\omega_{\lambda^{\prime \prime}}\right)\right\} .
\end{aligned}
$$

Here, $\lambda$ is a shorthand notation for (q, $s)$ and phonon momentum is conserved in the three-phonon processes above. The three phonon matrix element $\Phi_{\lambda^{\prime} \lambda^{\prime \prime}}$ is calculated using

$$
\Phi_{\lambda^{\prime} \lambda^{\prime \prime}}=\sum_{i j k} \sum_{\alpha \beta \gamma} \frac{X_{\lambda}^{i \alpha} X_{\lambda^{\prime}}^{j \beta} X_{\lambda^{\prime \prime}}^{k \gamma}}{\sqrt{m_{i} m_{j} m_{k}} \sqrt{\omega_{\lambda} \omega_{\lambda^{\prime}} \omega_{\lambda^{\prime \prime}}}} \Phi_{i j k}^{\alpha \beta \gamma} \mathrm{e}^{i \mathbf{q} \cdot \mathbf{r}_{i}+i \mathbf{q}^{\prime} \cdot \mathbf{r}_{j}+i \mathbf{q}^{\prime \prime} \cdot \mathbf{r}_{k}},
$$

where $m_{i}$ is the mass of atom $i, X_{\lambda}^{i a}$ is the component $a$ of the eigenvector for mode $\lambda$ and atom $i$, and $\mathbf{r}_{i}$ is the vector associated with atom $i$. $\omega_{\lambda}$ is the TDEP frequency of the phonon mode $\lambda$, and $\Phi_{i j k}^{a \beta \gamma}$ is the third-order interatomic force constant. This expression for the imaginary part of the self-energy is the result of the first-order perturbation theory for the phonon self-energy due to third-order anharmonicity (so-called bubble term in the diagrammatic representation of the self-energy). The real part of self-energy is the Kramers-Kronig transformation of the imaginary part. A sampling of phonon states is carried out using a $30 \times 30 \times 30$ qpoint grid.

\section{Calculation of structural parameters}

The temperature evolution of the crystal lattice of GeTe was calculated using MD. We ran MD calculations using the LAMMPS software ${ }^{78}$. To perform MD simulations, we developed a very accurate interatomic potential based on the Gaussian Approximation Potentials scheme ${ }^{79,80}$ (see Supplementary Note 6). To obtain structural parameters at the temperature $T$, we ran the NPT ensemble MD calculation of a 2000 atom cell (the $10 \times 10 \times 10$ supercells) fixing pressure to $0 \mathrm{~Pa}$ (we checked the convergence with respect to simulation cell size and found the 2000 atom cell to be converged). Following a 10 and 20 ps equilibration in the NVT and NPT ensembles respectively, we ran the simulation for 100 ps sampling the lattice constant $a$, the rhombohedral angle $\theta$ and the volume every $100 \mathrm{fs}$. For the calculation of the interatomic displacement parameter $\mu$, we sampled atomic positions every timestep ( $1 \mathrm{fs})$. Final structural parameters were obtained as a simple sample average and are given in Supplementary Note 7. We obtain negative thermal expansion at the phase transition ${ }^{41,65,67,81}$. In our MD simulations, the system is still ferroelectric at $631 \mathrm{~K}$, while it is paraelectric at $637 \mathrm{~K}$. Because of this, we define the critical temperature as the average of these two temperatures $\left(T_{\mathrm{C}}=634 \mathrm{~K}\right)$.

\section{TDEP method}

To get temperature-dependent interatomic force constants, we use the TDEP method ${ }^{34-36}$. This approach employs a fitting procedure of the second and third-order force constants to DFT forces on forces sampled along an MD trajectory. We ran MD simulations using the GAP potential in the NVT ensemble on the structures obtained from the MD study of thermal expansion to obtain atomic configurations. After $50 \mathrm{ps}$ of equilibration, we sampled 24 configurations on the $300 \mathrm{ps}$ long trajectory. We used a 512 atom supercell to converge phonon properties with respect to the second and third-order force constants cutoff (12 and $8 \AA$, respectively). We extracted selected configurations and carried out DFT calculations on them 
to obtain forces. Since the $0 \mathrm{~K}$ structures of GeTe obtained using GAP and DFT are slightly different (see Supplementary Note 6), we obtained the DFT structure of GeTe at a certain temperature using the thermal expansion coefficients calculated with MD combined with the $0 \mathrm{~K}$ DFT structure.

We note that we did not include LO/TO splitting due to long-range electrostatic forces in any of our calculations. GeTe has a large number of intrinsic vacancies which give large free charge carrier concentrations. Free charge carriers will perfectly screen the long-range interaction ${ }^{45}$, resulting in no LO/TO splitting in real samples.

\section{Density functional theory}

DFT calculations were performed using the ABINIT software package ${ }^{82,83}$. We use a generalised gradient approximation with the Perdew-Burke-Ernzerhof parametrization $^{84}$ for the exchange-correlation functional and the Hartwigsen-Goedecker-Hutter pseudopotentials ${ }^{85}$. Wave functions are represented in a plane wave basis set with the cutoff of $16 \mathrm{Ha}$, and the $\Gamma$ point is used for sampling of electronic states.

\section{DATA AVAILABILITY}

The authors declare that the data supporting the present work is available from the corresponding authors upon reasonable request. The GAP interatomic potential for $\mathrm{GeTe}$ is available from Materials Cloud repository ${ }^{86}$.

\section{CODE AVAILABILITY}

The code that implements the temperature effective potential (TDEP) method is available from Olle Hellman upon reasonable request. The additional data processing scripts and codes are available from the corresponding authors. GAP software is available for non-commercial use from www.libatoms.org.

Received: 15 December 2020; Accepted: 19 March 2021; Published online: 29 April 2021

\section{REFERENCES}

1. Tan, G., Zhao, L.-D. \& Kanatzidis, M. G. Rationally designing high-performance bulk thermoelectric materials. Chem. Rev. 116, 12123-12149 (2016).

2. Chang, $C$. et al. 3D charge and $2 \mathrm{D}$ phonon transports leading to high out-of-plane zT in n-type SnSe crystals. Science 360, 778-783 (2018).

3. Chen, $\mathrm{Z}$. et al. Lattice dislocations enhancing thermoelectric PbTe in addition to band convergence. Adv. Mater. 29, 1606768 (2017).

4. Biswas, K. et al. High-performance bulk thermoelectrics with all-scale hierarchical architectures. Nature 489, 414-418 (2012).

5. Zhao, L.-D. et al. Ultralow thermal conductivity and high thermoelectric figure of merit in SnSe crystals. Nature 508, 373-377 (2014).

6. Vineis, C. J., Shakouri, A., Majumdar, A. \& Kanatzidis, M. G. Nanostructured thermoelectrics: big efficiency gains from small features. Adv. Mater. 22, 3970-3980 (2010).

7. Beekman, M., Morelli, D. T. \& Nolas, G. S. Better thermoelectrics through glass-like crystals. Nat. Mater. 14, 1182-1185 (2015).

8. Tan, Q. et al. Thermoelectrics with earth abundant elements: low thermal conductivity and high thermopower in doped SnS. J. Mater. Chem. A 2, 17302-17306 (2014).

9. Dirmyer, M. R., Martin, J., Nolas, G. S., Sen, A. \& Badding, J. V. Thermal and electrical conductivity of size-tuned bismuth telluride nanoparticles. Small $\mathbf{5}$, 933-937 (2009).

10. Biswas, K., Zhao, L.-D. \& Kanatzidis, M. G. Tellurium-free thermoelectric: the anisotropic n-type semiconductor $\mathrm{Bi}_{2} \mathrm{~S}_{3}$. Adv. Energy Mater. 2, 634-638 (2012).

11. Hellman, O. \& Broido, D. A. Phonon thermal transport in $\mathrm{Bi}_{2} \mathrm{Te}_{3}$ from first principles. Phys. Rev. B 90, 134309 (2014).

12. Tse, J. S. et al. Anharmonic motions of $\mathrm{Kr}$ in the clathrate hydrate. Nat. Mater. 4, 917 (2005).

13. Avila, M. A. et al. $\mathrm{Ba}_{8} \mathrm{Ga}_{16} \mathrm{Sn}_{30}$ with type-I clathrate structure: drastic suppression of heat conduction. Appl. Phys. Lett. 92, 041901 (2008).

14. Christensen, M. et al. Avoided crossing of rattler modes in thermoelectric materials. Nat. Mater. 7, 811 (2008).

15. Tang, Y. et al. Convergence of multi-valley bands as the electronic origin of high thermoelectric performance in $\mathrm{CoSb}_{3}$ skutterudites. Nat. Mater. 14, 1223-1228 (2015).
16. Jana, M. K., Pal, K., Waghmare, U. V. \& Biswas, K. The origin of ultralow thermal conductivity in InTe: lone-pair-induced anharmonic rattling. Angew. Chem. Int. Ed. 55, 7792-7796 (2016).

17. Lory, P.-F. et al. Direct measurement of individual phonon lifetimes in the clathrate compound $\mathrm{Ba}_{7.81} \mathrm{Ge}_{40.67} \mathrm{Au}_{5.33}$. Nat. Commun. 8, 491 (2017).

18. Delaire, O. et al. Giant anharmonic phonon scattering in PbTe. Nat. Mater. 10, 614-619 (2011).

19. Shiga, T. et al. Microscopic mechanism of low thermal conductivity in lead telluride. Phys. Rev. B 85, 155203 (2012).

20. Li, C. W. et al. Phonon self-energy and origin of anomalous neutron scattering spectra in SnTe and PbTe thermoelectrics. Phys. Rev. Lett. 112, 175501 (2014).

21. Romero, A. H., Gross, E. K. U., Verstraete, M. J. \& Hellman, O. Thermal conductivity in PbTe from first principles. Phys. Rev. B 91, 214310 (2015).

22. Murphy, R. M., Murray, É. D., Fahy, S. \& Savić, I. Broadband phonon scattering in PbTe-based materials driven near ferroelectric phase transition by strain or alloying. Phys. Rev. B 93, 104304 (2016).

23. Murphy, R. M., Murray, É. D., Fahy, S. \& Savić, I. Ferroelectric phase transition and the lattice thermal conductivity of $\mathrm{Pb}_{1-x} \mathrm{Ge}_{x}$ Te alloys. Phys. Rev. B 95, 144302 (2017).

24. Xia, Y. \& Chan, M. K. Y. Anharmonic stabilization and lattice heat transport in rocksalt $\beta$-GeTe. Appl. Phys. Lett. 113, 193902 (2018).

25. Li, J. et al. Simultaneous optimization of carrier concentration and alloy scattering for ultrahigh performance GeTe thermoelectrics. Adv. Sci. 4, 1700341 (2017).

26. Perumal, S., Roychowdhury, S., Negi, D. S., Datta, R. \& Biswas, K. High thermoelectric performance and enhanced mechanical stability of p-type $\mathrm{Ge}_{1-x} \mathrm{Sb}_{x} \mathrm{Te}$. Chem. Mater. 27, 7171-7178 (2015).

27. Roychowdhury, S., Samanta, M., Perumal, S. \& Biswas, K. Germanium chalcogenide thermoelectrics: electronic structure modulation and low lattice thermal conductivity. Chem. Mater. 30, 5799-5813 (2018).

28. Xing, T. et al. Superior performance and high service stability for GeTe-based thermoelectric compounds. Natl. Sci. Rev. 6, 944-954 (2019).

29. Jin, Y. et al. Realizing high thermoelectric performance in GeTe through optimizing Ge vacancies and manipulating Ge precipitates. ACS Appl. Energy Mater. 2, 7594-7601 (2019).

30. Nshimyimana, E. et al. Discordant nature of $\mathrm{Cd}$ in GeTe enhances phonon scattering and improves band convergence for high thermoelectric performance. $J$. Mater. Chem. A 8, 1193-1204 (2020).

31. Liu, Z. et al. Phase-transition temperature suppression to achieve cubic GeTe and high thermoelectric performance by $\mathrm{Bi}$ and $\mathrm{Mn}$ codoping. Proc. Natl Acad. Sci. USA 115, 5332-5337 (2018).

32. $\mathrm{Li}$, J. et al. Stacking fault-induced minimized lattice thermal conductivity in the high-performance GeTe-based thermoelectric materials upon $\mathrm{Bi}_{2} \mathrm{Te}_{3}$ alloying. ACS Appl. Mater. Interfaces 11, 20064-20072 (2019).

33. Philip, J. Thermal conduction across ferroelectric phase transitions: results on selected systems. In (ed Lallart, M.) Ferroelectrics. chap. 7 (IntechOpen, Rijeka, 2011)

34. Hellman, O., Abrikosov, I. A. \& Simak, S. I. Lattice dynamics of anharmonic solids from first principles. Phys. Rev. B 84, 180301 (2011).

35. Hellman, O. \& Abrikosov, I. A. Temperature-dependent effective third-order interatomic force constants from first principles. Phys. Rev. B 88, 144301 (2013).

36. Hellman, O., Steneteg, P., Abrikosov, I. A. \& Simak, S. I. Temperature dependent effective potential method for accurate free energy calculations of solids. Phys. Rev. B 87, 104111 (2013).

37. Rinaldi, C. et al. Ferroelectric control of the spin texture in GeTe. Nano Lett. 18 2751-2758 (2018).

38. Krempaský, J. et al. Spin-resolved electronic structure of ferroelectric $a$-GeTe and multiferroic $\mathrm{Ge}_{1-x} \mathrm{Mn}_{x} \mathrm{Te}$. J. Phys. Chem. Solids 128, 237-244 (2019).

39. Di Sante, D., Barone, P., Bertacco, R. \& Picozzi, S. Electric control of the giant Rashba effect in bulk GeTe. Adv. Mater. 25, 509-513 (2013).

40. Liebmann, M. et al. Giant Rashba-type spin splitting in ferroelectric GeTe(111). Adv. Mater. 28, 560-565 (2016).

41. Chattopadhyay, T., Boucherle, J. X. \& vonSchnering, H. G. Neutron diffraction study on the structural phase transition in GeTe. J. Phys. C 20, 1431 (1987).

42. Chatterji, T., Kumar, C. M. N. \& Wdowik, U. D. Anomalous temperature-induced volume contraction in GeTe. Phys. Rev. B 91, 054110 (2015).

43. Fons, P. et al. Phase transition in crystalline GeTe: pitfalls of averaging effects Phys. Rev. B 82, 155209 (2010).

44. Matsunaga, T., Fons, P., Kolobov, A. V., Tominaga, J. \& Yamada, N. The orderdisorder transition in GeTe: views from different length-scales. Appl. Phys. Lett. 99, 231907 (2011).

45. Steigmeier, E. \& Harbeke, G. Soft phonon mode and ferroelectricity in GeTe. Solid State Commun. 8, 1275-1279 (1970).

46. Landau, L. \& Lifshitz, E. Statistical Physics. Vol. 5 (Elsevier Science, 2013).

47. Dougherty, T. P. et al. Femtosecond resolution of soft mode dynamics in structural phase transitions. Science 258, 770-774 (1992). 
48. Kadlec, F., Kadlec, C., Kužel, P. \& Petzelt, J. Study of the ferroelectric phase transition in germanium telluride using time-domain terahertz spectroscopy. Phys. Rev. B 84, 205209 (2011).

49. Abrikosov, N. K., Bankina, V. F., Poretskaya, L. V., Shelimova, L. E. \& Skudnova, E. V. Semiconducting II-VI, IV-VI, and V-VI Compounds. Monographs in Semiconductor Physics (Springer, USA, 1969).

50. Marchenkov, E. A. \& Shipul', V. P. Thermal expansion of semiconductor materials. J. Eng. Phys. Thermophys. 66, 547-551 (1994).

51. Maradudin, A. A. \& Fein, A. E. Scattering of neutrons by an anharmonic crystal. Phys. Rev. 128, 2589-2608 (1962).

52. Cowley, R. A. Anharmonic crystals. Rep. Prog. Phys. 31, 123-166 (1968),

53. Kokkedee, J. Anharmonic effects in the coherent scattering of neutrons by crystals: a formal treatment of shift and width of the peaks in the scattering spectrum. Physica 28, 374-408 (1962).

54. Cowley, R. A. The theory of raman scattering from crystals. Proc. Phys. Soc. 84, 281-296 (1964).

55. Li, C. W. et al. Orbitally driven giant phonon anharmonicity in SnSe. Nat. Phys. 11, 1063-1069 (2015).

56. Ribeiro, G. A. S. et al. Strong anharmonicity in the phonon spectra of $\mathrm{PbTe}$ and SnTe from first principles. Phys. Rev. B 97, 014306 (2018).

57. Aseginolaza, U. et al. Phonon collapse and second-order phase transition in thermoelectric SnSe. Phys. Rev. Lett. 122, 075901 (2019).

58. Aseginolaza, U. et al. Strong anharmonicity and high thermoelectric efficiency in high-temperature SnS from first principles. Phys. Rev. B 100, 214307 (2019).

59. Dangić, Đ, Murray, É. D., Fahy, S. \& Savić, I. Structural and thermal transport properties of ferroelectric domain walls in GeTe from first principles. Phys. Rev. B 101, 184110 (2020).

60. Lee, H. S. et al. Herringbone structure in GeTe-based thermoelectric materials. Acta Mater. 91, 83-90 (2015).

61. Vermeulen, P. A., Kumar, A., ten Brink, G. H., Blake, G. R. \& Kooi, B. J. Unravelling the domain structures in GeTe and $\mathrm{LaAlO}_{3}$. Cryst. Growth Des. 16, 5915-5922 (2016).

62. Nukala, P. et al. Inverting polar domains via electrical pulsing in metallic germanium telluride. Nat. Commun. 8, 15033 EP- (2017).

63. Polking, M. J., Zheng, H., Ramesh, R. \& Alivisatos, A. P. Controlled synthesis and size-dependent polarization domain structure of colloidal germanium telluride nanocrystals. J. Am. Chem. Soc. 133, 2044-2047 (2011).

64. Campi, D., Paulatto, L., Fugallo, G., Mauri, F. \& Bernasconi, M. First-principles calculation of lattice thermal conductivity in crystalline phase change materials: GeTe, $\mathrm{Sb}_{2} \mathrm{Te}_{3}$, and $\mathrm{Ge}_{2} \mathrm{Sb}_{2} \mathrm{Te}_{5}$. Phys. Rev. B 95, 024311 (2017).

65. Sist, M., Kasai, H., Hedegaard, E. M. J. \& Iversen, B. B. Role of vacancies in the hightemperature pseudodisplacive phase transition in GeTe. Phys. Rev. B 97, 094116 (2018).

66. Callaway, J. Model for lattice thermal conductivity at low temperatures. Phys. Rev. 113, 1046-1051 (1959).

67. Dangić, Đ, Murphy, A. R., Murray, É. D., Fahy, S. \& Savić, I. Coupling between acoustic and soft transverse optical phonons leads to negative thermal expansion of GeTe near the ferroelectric phase transition. Phys. Rev. B 97, 224106 (2018).

68. Srivastava, G. The Physics of Phonons (Taylor \& Francis, 1990).

69. Semwal, B. S. \& Sharma, P. K. Thermal conductivity of an anharmonic crystal. Phys. Rev. B 5, 3909-3914 (1972).

70. Hardy, R. J. Energy-flux operator for a lattice. Phys. Rev. 132, 168-177 (1963).

71. Horie, C. \& Krumhansl, J. A. Boltzmann equation in a phonon system. Phys. Rev. 136, A1397-A1407 (1964)

72. Kubo, R. Statistical-mechanical theory of irreversible processes. I. General theory and simple applications to magnetic and conduction problems. J. Phys. Soc. Jpn 12, 570-586 (1957).

73. Pathak, K. N. Theory of anharmonic crystals. Phys. Rev. 139, A1569-A1580 (1965).

74. Deo, B. \& Behera, S. N. Calculation of thermal conductivity by the Kubo formula. Phys. Rev. 141, 738-741 (1966).

75. Zubarev, D. N. Double-time Green functions in statistical physics. Sov. Phys. Uspekhi 3, 320-345 (1960).

76. Simoncelli, M., Marzari, N. \& Mauri, F. Unified theory of thermal transport in crystals and glasses. Nat. Phys. 15, 809-813 (2019).

77. Isaeva, L., Barbalinardo, G., Donadio, D. \& Baroni, S. Modeling heat transport in crystals and glasses from a unified lattice-dynamical approach. Nat. Commun. 10, 3853 (2019).

78. Plimpton, S. Fast parallel algorithms for short-range molecular dynamics. J. Comput. Phys. 117, 1-19 (1995).
79. Bartók, A. P., Payne, M. C., Kondor, R. \& Csányi, G. Gaussian approximation potentials: the accuracy of quantum mechanics, without the electrons. Phys. Rev. Lett. 104, 136403 (2010)

80. Bartók, A. P., Kondor, R. \& Csányi, G. On representing chemical environments. Phys. Rev. B 87, 184115 (2013).

81. Gainza, J. et al. Features of the high-temperature structural evolution of GeTe thermoelectric probed by neutron and synchrotron powder diffraction. Metals $\mathbf{1 0}$ (2020).

82. Gonze, X. et al. Abinit: First-principles approach to material and nanosystem properties. Comput. Phys. Commun. 180, 2582-2615 (2009).

83. Gonze, X. et al. Recent developments in the abinit software package. Comput. Phys. Commun. 205, 106-131 (2016).

84. Perdew, J. P., Burke, K. \& Ernzerhof, M. Generalized gradient approximation made simple. Phys. Rev. Lett. 77, 3865-3868 (1996).

85. Goedecker, S., Teter, M. \& Hutter, J. Separable dual-space gaussian pseudopotentials. Phys. Rev. B 54, 1703-1710 (1996).

86. Dangić, Đ., Fahy, S. \& Savić, I. Gaussian approximation potentials (GAP) for germanium telluride. Mater. Cloud Archive 2021.42, (2021).

\section{ACKNOWLEDGEMENTS}

This work is supported by Science Foundation Ireland under grant numbers 15/IA/ 3160 and 13/RC/2077. The later grant is co-funded under the European Regional Development Fund. O.H. gratefully acknowledges support from the Knut and Alice Wallenberg Foundation (Wallenberg Scholar Grant No. KAW-2018.0194) and the Swedish Government Strategic Research Areas SeRC. We acknowledge the Irish Centre for High-End Computing (ICHEC) for the provision of computational facilities.

\section{AUTHOR CONTRIBUTIONS}

I.S. and Đ.D. conceived the research plan. Đ.D. performed the calculations. I.S. supervised the work. Đ.D. and I.S. wrote the manuscript with contribution from all authors. O.H. provided the code for the TDEP method. All authors discussed and interpreted the results.

\section{COMPETING INTERESTS}

The authors declare no competing interests.

\section{ADDITIONAL INFORMATION}

Supplementary information The online version contains supplementary material available at https://doi.org/10.1038/s41524-021-00523-7.

Correspondence and requests for materials should be addressed to Đ.D. or I.S.

Reprints and permission information is available at http://www.nature.com/reprints

Publisher's note Springer Nature remains neutral with regard to jurisdictional claims in published maps and institutional affiliations.

Open Access This article is licensed under a Creative Commons Attribution 4.0 International License, which permits use, sharing, adaptation, distribution and reproduction in any medium or format, as long as you give appropriate credit to the original author(s) and the source, provide a link to the Creative Commons license, and indicate if changes were made. The images or other third party material in this article are included in the article's Creative Commons license, unless indicated otherwise in a credit line to the material. If material is not included in the article's Creative Commons license and your intended use is not permitted by statutory regulation or exceeds the permitted use, you will need to obtain permission directly from the copyright holder. To view a copy of this license, visit http://creativecommons. org/licenses/by/4.0/.

(c) The Author(s) 2021 